\title{
A statistically significant clustering
}

Astron. J. 157, 62 (2019)

The existence of a massive planet at the outskirts of the Solar System, commonly called Planet Nine, is currently one of the most debated issues in planetary science. The Planet Nine hypothesis stemmed from observations that showed that the orbits of the most distant trans-Neptunian objects (TNOs) are not randomly distributed but instead clustered in space. However, the existence of such clustering has also been attributed to observational bias or to the small sample of objects. Michael Brown and Konstantin Batygin employ a statistical analysis of all the known distant TNOs to show that such clustering has a very high probability to be real.

Brown and Batygin determine the possible observational biases on the argument of perihelion and the position of the orbital pole for the 14 known objects with semi-major axis beyond $230 \mathrm{au}$. Then they combine them in order to reconstruct the probability distribution that each object would have been discovered at a specific position, under the assumption that there is no real clustering and the spatial distribution of TNOs is uniform. Finally, they perform 1.6 million iterations by randomizing the combined position of all 14 TNOs at each iteration. They find that only $0.2 \%$ of the runs could reproduce the observed position of all 14 objects, under the uniform distribution assumption.

Brown and Batygin also discuss the recent results from the OSSOS survey, which found four distant TNOs whose positions seem to be consistent with a uniform distribution (C. Shankman et al. Astron. J. 154, 50; 2017), showing that it cannot reach any definite conclusion on the clustering properties because of its significant longitudinal bias.

Brown and Batygin stay agnostic concerning the cause of the clustering even though they remark that Planet Nine is currently the only hypothesis that can explain all the observations.

Luca Maltagliati

Published online: 27 February 2019

https://doi.org/10.1038/s41550-019-0732-0 